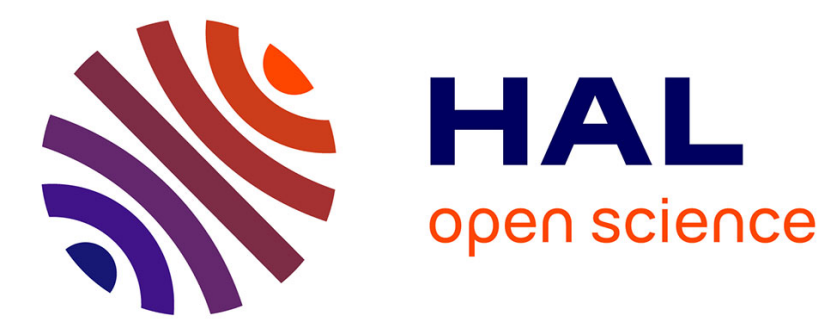

\title{
LES USAGES SOCIAUX DES EMPLOIS PRÉCAIRES DANS LES INSTITUTIONS CULTURELLES
}

\author{
Aurélie Peyrin
}

\section{To cite this version:}

Aurélie Peyrin. LES USAGES SOCIAUX DES EMPLOIS PRÉCAIRES DANS LES INSTITUTIONS

CULTURELLES: Le cas des médiateurs de musées. Sociétés contemporaines, 2007, 3 (67), p. 7-26. 10.3917/soco.067.0007 . halshs-01102533

\section{HAL Id: halshs-01102533 \\ https://shs.hal.science/halshs-01102533}

Submitted on 13 Jan 2015

HAL is a multi-disciplinary open access archive for the deposit and dissemination of scientific research documents, whether they are published or not. The documents may come from teaching and research institutions in France or abroad, or from public or private research centers.
L'archive ouverte pluridisciplinaire HAL, est destinée au dépôt et à la diffusion de documents scientifiques de niveau recherche, publiés ou non, émanant des établissements d'enseignement et de recherche français ou étrangers, des laboratoires publics ou privés. 


\section{LES USAGES SOCIAUX DES EMPLOIS PRÉCAIRES DANS LES INSTITUTIONS CULTURELLES.}

Le cas des médiateurs de musées

Aurélie Peyrin

Presses de Sciences Po | Sociétés contemporaines

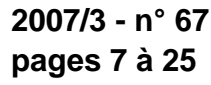

ISSN 1150-1944

Article disponible en ligne à l'adresse:

http://www.cairn.info/revue-societes-contemporaines-2007-3-page-7.htm

Pour citer cet article :

Peyrin Aurélie, « Les usages sociaux des emplois précaires dans les institutions culturelles. » Le cas des médiateurs de musées,

Sociétés contemporaines, 2007/3 n67, p. 7-25. DOI : 10.3917/soco.067.0007

Distribution électronique Cairn.info pour Presses de Sciences Po.

(C) Presses de Sciences Po. Tous droits réservés pour tous pays.

La reproduction ou représentation de cet article, notamment par photocopie, n'est autorisée que dans les limites des conditions générales d'utilisation du site ou, le cas échéant, des conditions générales de la licence souscrite par votre établissement. Toute autre reproduction ou représentation, en tout ou partie, sous quelque forme et de quelque manière que ce soit, est interdite sauf accord préalable et écrit de l'éditeur, en dehors des cas prévus par la législation en vigueur en France. II est précisé que son stockage dans une base de données est également interdit. 


\section{Aurélie Peyrin}

\section{LES USAGES SOCIAUX DES EMPLOIS PRÉCAIRES DANS LES INSTITUTIONS CULTURELLES. LE CAS DES MÉDIATEURS DE MUSÉES}

Résumé : L'analyse des usages sociaux des formes particulières d'emploi public permet de mettre en relief les ajustements et désajustements entre logiques de segmentation des employeurs d'une part, et profils et stratégies des salariés, d'autre part. En prenant l'exemple de l'accompagnement des visiteurs dans les musées territoriaux, cet article montre que le système de gestion flexible qui repose sur un recours massif aux médiateurs vacataires semble convenir à certains profils, les étudiants ou les artistes par exemple. Mais, pour durer dans le métier, la plupart des médiateurs vacataires complètent les revenus de l'activité d'accompagnement dans un musée par des revenus tirés de la même activité pour un autre employeur. En diminuant la disponibilité des médiateurs, cette stratégie perturbe l'organisation muséale et incite les employeurs à imaginer d'autres modes de gestion de la main d'œuvre d'accompagnement.

)

epuis les années 1980, les politiques publiques en faveur de la démocratisation culturelle ont favorisé le développement de services d'accompagnement des visiteurs dans les musées sous tutelle de l'État. À travers les visites guidées, les conférences ou les ateliers confiés à des médiateurs, les collectivités publiques cherchent à diversifier la composition sociale des visiteurs de leurs musées, en attirant ceux qui n'y viennent pas : les moins diplômés, membres des professions et catégories socioprofessionnelles des ouvriers et employés (Donnat, 1999). L'objectif de réduction des inégalités sociales d'accès à la culture a été réaffirmé par la loi $\mathrm{n}^{\circ}$ 2002-5 du 4 janvier 2002 relative aux musées de France, qui inscrit les «actions d'éducation et de diffusion visant à assurer l'égal accès de tous à la culture » parmi les missions permanentes des musées de France (Peyrin, 2005).

Cette loi constitue le point d'orgue de trois décennies durant lesquelles les musées français se sont transformés (Ballé et Poulot, 2005), et à l'issue desquelles les professionnels les plus « emblématiques » des musées, leurs conservateurs, sont devenus un objet sociologique. Les chercheurs se sont intéressés à ce groupe professionnel sous plusieurs angles: en tant qu'acteurs de la constitution de valeurs sur le marché de l'art (Moulin et Quemin, 1993), ou d'un 
processus de professionnalisation (Octobre, 2001). Plus récemment, l'accent a été mis sur le travail des conservateurs et leur rôle dans la mise en œuvre du service public (Join-Lambert et al., 2004 ; Poulard, 2005). Dans ces recherches, le groupe professionnel des médiateurs est parfois évoqué car il participerait d'une remise en cause de la division du travail établie au sein des musées, en contestant notamment la légitimité des conservateurs à diriger ces établissements (Join-Lambert et al., 2004).

Le groupe professionnel des médiateurs s'inscrit bien, lui aussi, dans des problématiques de professionnalisation et de division du travail, mais ses caractéristiques atypiques ouvrent d'autres questionnements relatifs à la flexibilité et à la précarité, appliqués au terrain de l'emploi public (Fons et Meyer, 2005 ; Berg et al., 2006). Alors qu'ils sont généralement diplômés de l'enseignement supérieur long comme les conservateurs, 70 \% des médiateurs sont employés de façon précaire, sur des formes particulières d'emploi que la fonction publique rassemble sous l'appellation de «non titulaires». Depuis l'invention d'une offre d'accompagnement pour les visiteurs des musées nationaux au début du siècle, ce travail (visites guidées) a été confié à de jeunes diplômées de l'École du Louvre, en majorité des femmes, qui travaillaient comme vacataires. Ce système flexible fonctionne grâce à un réservoir de main d'œuvre constitué par les étudiants et diplômés en histoire de l'art (ou toute autre discipline correspondant aux collections du musée : ethnologie, biologie, physique, etc.). Dans les années 2000, ces étudiants et jeunes diplômés sont en majorité des femmes (environ deux tiers à l'échelle des musées de France), généralement recrutés à partir de la Licence (bac plus trois), à l'issue d'un stage.

Pourquoi, alors qu'il a toujours été conçu comme le bras armé des politiques publiques de démocratisation culturelle - et donc comme relevant du service public -, l'accompagnement des visiteurs dans les musées a-t-il toujours été massivement pris en charge par des vacataires, salariés publics payés à la tâche? Et pourquoi ces pratiques perdurent-elles aujourd'hui, alors que la demande de services d'accompagnement semble augmenter sans cesse? Cet article explore deux pistes explicatives étroitement mêlées: les usages sociaux des formes particulières d'emploi, par les employeurs d'une part et par les salariés, d'autre part. On s'intéresse donc à la fois à la flexibilité du travail et à la flexibilité de l'emploi, en étudiant la façon dont les stratégies de gestion de la main d'œuvre déterminent l'organisation du travail. La flexibilité des emplois n'apporte pas toujours une flexibilité optimale dans l'organisation du travail ; certains profils de médiateurs peuvent s'accommoder de conditions précaires. Ces deux hypothèses sont englobées dans une problématique d'ajustements et de désajustements 
entre les usages propres à chaque type d'acteurs, logiques d'employeurs (segmentation de l'emploi) et caractéristiques de l'offre de travail (profils et trajectoires biographiques) interagissant constamment.

\section{DONNÉES ET MÉTHODES}

L'enquête exploitée ici combine deux types de matériaux pour réaliser le portrait des médiateurs de musées à travers le prisme du travail, de l'emploi et de l'identité professionnelle (Peyrin, 2005). Le volet qualitatif fournit la matière d'une analyse des trajectoires contextualisées par des études d'organisations, tandis que le volet quantitatif permet de tester statistiquement la pertinence de ces modèles organisationnels pour fournir un cadre général à l'échelle des musées français sous tutelle du ministère de la Culture et de la Communication.

L'enquête qualitative a été conduite en 2002 et 2003 dans une vingtaine de musées aux collections variées, situés en région Île-de-France, Alsace et RhôneAlpes et essentiellement financés par des fonds publics /collectivités territoriales et/ou État). Elle comportait des séances d'observation du travail et des interactions au sein du musée et du service des publics, enrichies par une centaine d'entretiens approfondis - dont les deux tiers auprès de médiateurs. Pour cette population qui constitue le cœur de l'enquête, les entretiens biographiques portaient sur la formation, l'insertion professionnelle et les questions statutaires, le travail et les relations de travail au quotidien. La partie quantitative a été réalisée en 2004 ; elle prenait la forme d'une exploitation secondaire du recensement administratif des personnels et moyens affectés à l'accompagnement des visiteurs dans les musées de France (sous tutelle du ministère de la Culture), réalisée par la Direction des Musées de France (DMF) en 2001 . Le questionnaire adressé à quelque 1300 établissements a été rempli et retourné par $54 \%$ d'entre eux ${ }^{2}$. Au final, la base de données comprend 709 musées au sein desquels l'effectif du service des publics est précisément renseigné ${ }^{3}: 1948$ personnes étaient recensées dans les musées répondants (dont 936 médiateurs), pour lesquelles on dispose d'informations relatives au sexe, à la fonction (poste occupé), au statut de l'emploi et au temps de travail. Aucune information relative à l'âge, au diplôme, au salaire ou à la trajectoire antérieure ne figure en revanche dans cette base administrative.

La première partie présente quelques éléments de cadrage sur le recours aux formes d'emploi précaires dans la fonction publique territoriale, pour comprendre la mise en place d'une gestion flexible du travail d'accompagnement des visiteurs dans les musées. Plus des deux tiers des médiateurs sont en emploi non permanent et à temps non complet, et $40 \%$ d'entre eux sont précisément vacataires, c'està-dire employés à la demande ${ }^{4}$. La seconde partie présente ensuite les ajustements entre usages des formes d'emploi précaires par les

\footnotetext{
2/ Les établissements répondants sont représentatifs de la population mère sur les critères suivants : statut du musée, taille du musée et taille de ville d'implantation, genre des collections.

3/ Parmi ces musées, 441 déclarent avoir un service des publics, soit $62 \%$. Mais seulement 381 de ces services ont un effectif égal ou supérieur à une personne équivalent temps plein, soit 54 \% des musées.

4/ Source: enquête Direction des Musées de France, 2001.
} 
employeurs et stratégies des salariés: dans certains cas, on peut observer des appariements entre une organisation du travail fondée sur la flexibilité et une utilisation des vacations comme emploi d'attente ou d'appoint. Le recours aux vacations fonctionne non seulement comme un filtre pour les candidats à l'emploi, mais il détermine aussi la composition et le fonctionnement du service dédié à l'accompagnement. La troisième partie aborde enfin les désajustements entre usages des vacations par les employeurs et par les salariés, c'est-à-dire des situations où cette politique d'emploi ne répond plus aux attentes des deux types d'acteurs. Les médiateurs confrontés à une précarité durable de leur emploi élaborent des stratégies de réduction de l'incertitude qui perturbent la flexibilité des organisations, et incitent les employeurs à reconsidérer leur mode de gestion de la main d'œuvre d'accompagnement.

\section{L'ACCOMPAGNEMENT MUSÉAL, UN EXEMPLE DE GESTION FLEXIBLE DES EMPLOIS DANS LA FONCTION PUBLIQUE TERRITORIALE}

L'accompagnement des visiteurs dans les musées est né au début du $x^{e}$ siècle dans les musées nationaux. Dès cette époque, il a été confié à des vacataires, recrutés parmi les étudiants et diplômés - et surtout les étudiantes et diplômées - en histoire de l'art, notamment de l'École du Louvre. Ce système d'emploi flexible permettait aux administrations publiques gestionnaires du personnel muséal de ne pas considérer les médiateurs comme leurs salariés, mais comme des intervenants ponctuels rémunérés à la tâche. Près d'un siècle plus tard, l'offre d'accompagnement est largement répandue dans l'ensemble des musées publics, majoritairement territoriaux, et le recours aux vacations demeure le mode dominant d'emploi des médiateurs.

Si l'injonction politique de démocratisation vient encore aujourd'hui de l'État, la majorité des médiateurs est recrutée et employée par les collectivités locales. Or, loin de présenter des caractéristiques distinctes du reste de l'économie, les institutions culturelles financées par les collectivités publiques sont confrontées aux mêmes défis et aux mêmes enjeux que les organisations du secteur privé. Appartenant à la fois aux secteurs culturels et touristiques, les musées doivent faire face à une demande instable, quoique globalement saisonnière. Dès les années 1940-1950, la population scolaire est en effet devenue la principale cliente de services d'accompagnement, sous forme de visites guidées, de visites animées ou d'ateliers. L'organisation du travail d'accompagnement dans les musées s'est donc progressivement alignée sur le 
calendrier scolaire, avec des périodes creuses pendant les vacances - ce qui est encore le cas aujourd'hui. De plus, la présence des tutelles publiques et la part prépondérante des subventions dans les crédits de fonctionnement incitent les musées à gérer leurs ressources humaines en s'efforçant de minimiser les coûts, d'autant que les fonctions de gestion proprement dites s'y développent (Tobelem, 2005).

À ces enjeux financiers et gestionnaires, les collectivités publiques répondent en segmentant leur main d'œuvre, selon des pratiques généralement présentées comme propres aux entreprises privées (Petit, 2005). Dans les musées dont elles sont propriétaires, les collectivités publiques n'emploient ainsi pas seulement des fonctionnaires, mais également de nombreux emplois précaires, dits « non titulaires », qui font office de variable d'ajustement aux besoins de flexibilité des organisations muséales. Les collectivités publiques sont autorisées par la loi à recruter des agents sur des formes particulières d'emploi, sous certains conditions : remplacer des titulaires à temps partiel ou en congé, combler des besoins occasionnels ou saisonniers, en cas de vacance de poste ou en l'absence d'un corps ou cadre d'emploi correspondant à la mission remplie par l'agent ${ }^{5}$. Les collectivités territoriales disposent ainsi d'une véritable palette contractuelle pour composer leurs stratégies de gestion de la main d'œuvre. Dans les musées, cette diversité statutaire concerne à la fois les médiateurs et les autres membres des services des publics, c'est-à-dire des équipes chargées de l'accompagnement des visiteurs (tableau 1).

\section{TABLEAU 1}

Distribution des membres des services des publics par forme d'emploi

\begin{tabular}{|c|c|c|c|c|}
\hline & \multicolumn{2}{|c|}{ Ensemble du service des publics } & \multicolumn{2}{|c|}{ Médiateurs } \\
\hline & \# & $\%$ & $\#$ & $\%$ \\
\hline Contrat à durée indéterminée & 34 & 2 & 23 & 3 \\
\hline Fonctionnaire & 532 & 35 & 177 & 26 \\
\hline Total emploi stable & 566 & 37 & 200 & 29 \\
\hline Vacataire & 326 & 21 & 279 & 40 \\
\hline Contrat à durée déterminée & 111 & 7 & 63 & 9 \\
\hline Emploi aidé & 267 & 17 & 146 & 21 \\
\hline Total emploi instable & 704 & 46 & 488 & 70 \\
\hline Professeurs mis à disposition & 259 & 17 & 5 & 1 \\
\hline Total & 1529 & 100 & 693 & 100 \\
\hline
\end{tabular}

Source : enquête DMF, 2001 (non réponses et autres réponses exclues).

5/ Le recours à des agents non titulaires (contractuels, auxiliaires, vacataires ou emplois aidés) dans la fonction publique française n'est pas nouveau, mais les sociologues et économistes s'y sont peu intéressés avant que des équipes françaises s'engagent dans des projets de comparaisons internationales (Berg et al., 2006). La recherche de flexibilité dans les administrations et structures relevant du service public est en effet un problème commun à l'ensemble des pays européens, même si la segmentation des emplois entre un noyau stable, une périphérie et des marges se décline de façon nettement différenciée d'un pays à l'autre (Fons et Meyer, 2005). 
Les contractuels et autres vacataires ont en commun un statut dérogatoire au statut général de la fonction publique (Aubin, 2004) : ce sont des emplois "sans statut», selon la formule de Dominique Schnapper (1989), qu'on peut considérer comme des formes particulières d'emploi propres au secteur public. Dans le système des vacations, le risque lié à l'activité, notamment le risque de l'emploi, est entièrement porté par le travailleur : " il s'agit pour les entreprises de se débarrasser de leurs obligations en matière de couverture sociale, d'assurance chômage, d'échapper à certaines réglementations et/ou d'extérioriser le risque de gestion de l'emploi ». (Dupuy et Larré, 1998, p. 12). Les vacataires sont considérés par le droit du travail comme des salariés puisque leurs employeurs et eux-mêmes sont assujettis aux cotisations de sécurité sociale et de retraite, mais le degré de protection des vacataires est proportionnel à leur activité. En outre, les vacataires et leurs employeurs ne cotisent pas au système d'assurance chômage. Leur protection est ainsi nettement inférieure à celle des salariés de droit commun et des agents publics (Moniolle, 1999).

Depuis la mise en place de la filière culturelle territoriale en 1991, trois nouveaux cadres d'emploi sont susceptibles d'accueillir les médiateurs: attaché de conservation du patrimoine (catégorie A), assistant et assistant qualifié de conservation du patrimoine (catégorie B). Les conditions de recours aux emplois non titulaires ne sont donc théoriquement plus aussi souples en ce qui les concerne, mais les pratiques traditionnelles persistent. Certains employeurs mettent par exemple en avant le caractère occasionnel de l'activité, alors même qu'ils emploient les mêmes personnes à ce poste depuis parfois plus de dix ans et ce, tout au long de l'année.

Nous allons à présent nous intéresser au fonctionnement des services des publics muséaux, en considérant d'abord les cas d'ajustements entre usages des formes particulières d'emploi par les employeurs et par les salariés. Nous montrerons que ce modèle d'emploi hybride, qui repose sur une répartition atypique des responsabilités et une reconsidération des engagements entre employeurs et employés, n'est pas sans conséquences sur la division du travail au sein des organisations muséales.

\section{DES PROFILS ADAPTÉS À UNE FLEXIBILITÉ ORGANISATIONNELLE?}

La tradition de recours à des médiateurs vacataires, forgée entre les années 1920 et 1940, s'est perpétuée au fil des années en raison 
d'une pratique de l'accompagnement caractérisée par l'intermittence ; elle a parallèlement été renforcée par la faiblesse statutaire de la fonction publique territoriale, lors de la diffusion de l'accompagnement dans les musées gérés par les collectivités territoriales entre les années 1970 et 1990. Cette pratique de la flexibilité a progressivement entraîné deux types de conséquences, qui contribuent à enfermer les organisations et les acteurs dans un recours quasi exclusif aux vacations pour l'accompagnement des visiteurs.

Première conséquence, les candidats aux fonctions d'accompagnement sont, hier comme aujourd'hui, ceux et celles qui « acceptent » un emploi non permanent et intermittent. Les médiateurs de musées sont essentiellement des jeunes femmes ${ }^{6}$, étudiantes ou récemment diplômées ${ }^{7}$, qui recherchent une première expérience de travail dans les musées, ou alors des artistes qui souhaitent un engagement rémunérateur mais peu contraignant pour préserver leur activité artistique. Seconde conséquence, les organisations muséales ont constitué des services des publics, dont la principale fonction consiste à apparier les demandes de services d'accompagnement par les clients (des établissements scolaires principalement, mais aussi des associations ou des individuels) à l'offre de travail des médiateurs, généralement limitée à quelques heures par semaine.

\section{LES VACATIONS COMME FILTRE POUR LES CANDIDATS AUX FONCTIONS D'ACCOMPAGNEMENT}

La plupart des médiateurs rencontrés au cours de l'enquête sont des enfants de la démocratisation universitaire, diplômés de nombreuses filières à dominante artistique ou culturelle. Une majorité d'entre eux sont des femmes, âgées de 25 à 45 ans, diplômées d'une maîtrise d'histoire de l'art ou d'un diplôme supérieur (nombre d'entre elles ayant mené en parallèle des études à l'École du Louvre). Les cursus universitaires de premier et second cycle comportant désormais des stages obligatoires, même dans les filières d'humanités classiques, de nombreux étudiants et étudiantes réalisent un stage dans les musées dans un cadre universitaire, soit dans les services des publics, soit dans les services de conservation des ouvres ou d'exposition $^{8}$. C'est souvent à cette occasion que sont proposées les premières vacations d'accompagnement, comme l'explique Audrey,

6/ Selon l'enquête de la Direction des Musées de France, $63 \%$ sont des femmes et $26 \%$ des hommes; pour $11 \%$ des individus, le sexe n'était pas renseigné.

7/ D’après l'enquête qualitative, le diplôme est généralement supérieur à la Licence (bac plus trois).

8/ Dans sa thèse consacrée aux conservateurs de musées territoriaux, Frédéric Poulard (2005) souligne lui aussi le rôle des étudiants stagiaires dans le travail de conservation. 
médiatrice contractuelle dans un musée d'art alsacien. "On a souvent des stagiaires qui travaillent avec la conservation, sur le petit journal de l'exposition. Donc nous, après, on les intègre pour les "fiches salle", parce qu'ils connaissent bien... [...] Après on les intègre en visites guidées, s'ils le veulent, donc ça leur permet de faire des vacations, et d'avoir une rémunération pour leur stage, finalement. »

De nombreux médiateurs ont ainsi commencé à exercer leur travail en cours d'études, à partir de la maîtrise ou du DEA, ou encore durant la thèse. Peggy par exemple, est doctorante en histoire de l'art; elle a 28 ans. Après un stage d'un an dans un grand musée d'art de la région Rhône-Alpes, elle a accepté les vacations d'accompagnement proposées par le responsable du service des publics parce qu'elle souhaitait continuer ses études (elle était alors en DEA) et que ses parents "n'arrivaient plus à suivre ». Elle considère ce travail uniquement comme un emploi d'attente, qui présente l'avantage d'être tout à fait cohérent avec ses études : " De toutes façons moi j'ai toujours pris mon travail d'animatrice comme un moyen de financer mes études. [...] J'ai eu la chance de financer mes études dans un domaine de mon choix, je trouve ça vachement bien, j'aurais pu être caissière à Carrefour, ça aurait été catastrophique.»

Le travail d'accompagnement est en effet décrit comme épanouissant, car il implique de conduire en permanence des recherches sur les œuvres et les artistes.
Le travail d'accompagnement est en effet décrit comme épanouissant, car il implique de conduire en permanence des recherches sur les œuvres et les artistes ; le contact du public est en outre considéré comme source d' « enrichissement personnel », et la mission de réduction des inégalités d'accès à la culture est enfin socialement valorisante. La précarité des emplois est par ailleurs compensée par la flexibilité du travail, qui permet aux médiateurs de maîtriser leur emploi du temps pour consacrer une partie de la semaine, du mois ou de l'année à d'autres activités (études, pratiques artistiques, famille, etc.). Ces jeunes adultes ne sont (ou ne pensent être à ce moment) médiateurs vacataires que temporairement, et ce n'est donc pas dans ce travail qu'ils projettent leur avenir ni qu'ils construisent leur identité (Fullin, 2005) : les vacations représentent une première marche dans un projet biographique et professionnel conçu par étapes, qui doit les mener vers un autre métier (Cancé, 2002). Peggy veut ainsi devenir enseignante-chercheuse ou conservatrice, et n'envisage en aucun cas de rester médiatrice après sa soutenance.

L'ajustement du profil des médiateurs aux formes particulières d'emploi caractérise aussi la situation des artistes. On retrouve dans leur cas la même dissociation entre l'activité de travail (l'accompagnement conçu comme "petit boulot alimentaire ») et 
activité extérieure au travail (l'activité créatrice considérée comme fondement de l'identité). Comme l'explique Ludovic, "mon métier premier c'est artiste, avec toute la précarité qui est scotchée à ce métier. Je ne vois pas forcément mon avenir ici, je me vois mal à cinquante ans être médiateur. Je vois ça comme un passage, même si ça peut être un long passage, j'espère être là plusieurs années encore, mais [...] ma priorité c'est la création, et puis continuer à trouver des boulots alimentaires...». Ludovic a 32 ans, il a été vacataire pendant deux ans dans le musée d'art alsacien qui l'emploie actuellement avant d'obtenir un contrat à durée déterminée. Il a néanmoins demandé à travailler à temps partiel pour préserver le temps consacré à la création. En entretien, il explique "pouvoir [se] le permettre ", car il vit avec sa conjointe, professeur des écoles titulaires. Les artistes qui gagnent leur vie comme médiateur vacataire bénéficient souvent d'arrangements conjugaux : dans le schéma de division sexuée du travail social [Breen et Cooke, 2005], ils occupent le rôle « féminin ». Ce schéma persiste évidemment parmi les jeunes femmes: Audrey, 28 ans, est médiatrice contractuelle dans le même musée que Ludovic, et a souhaité travailler à temps partiel pour consacrer du temps à ses deux jeunes enfants tout en conservant une activité professionnelle qu'elle apprécie.

L'offre d'emploi ne contribue pas seulement à attirer (et retenir) les candidats dont le profil et les projets peuvent s'accommoder d'un emploi du temps flexible et de faibles revenus mensuels, nous allons voir à présent qu'elle détermine aussi l'organisation du travail dans les services des publics.

\section{LES VACATIONS COMME RESSORT DE L'ORGANISATION DES SERVICES DES PUBLICS}

La moitié des musées de France ayant répondu à l'enquête de la Direction des Musées de France en 2001 dispose d'un « service des publics » comprenant au moins une personne équivalent temps plein ; l'effectif moyen de ces services est d'environ trois personnes. On trouve donc dans les musées de véritables équipes d'accompagnement, comprenant plusieurs types de postes en plus des médiateurs (tableau 2). Une liste exhaustive des fonctions rencontrées comprend : des agents de support, chargés de l'organisation pratique et administrative (accueil, réservations, secrétariat), des responsables de service chargés du pilotage de l'équipe, de la programmation de l'offre et des relations internes et externes; et enfin des enseignantsrelais, mis à disposition des musées quelques heures par semaine 
par le ministère de l'Éducation nationale ${ }^{9}$. La fonction d'accompagnement elle-même, qui correspond au poste de médiateur, occupe en moyenne une personne sur deux dans un service des publics: c'est le noyau fonctionnel des services des publics, puisque les médiateurs sont les prestataires directs des services aux visiteurs.

\section{TABLEAU 2}

Distribution des membres des services des publics des musées de France par poste de travail

\begin{tabular}{lcc} 
& $\#$ & $\%$ \\
\hline Médiateur & 936 & 55 \\
Professeur-relais & 251 & 15 \\
Chargé d'accueil & 191 & 11 \\
Responsable de service & 166 & 10 \\
Agent de support & 147 & 9 \\
Total & $\mathbf{1 6 9 1}$ & $\mathbf{1 0 0}$ \\
\hline
\end{tabular}

Source : enquête DMF, 2001.

Dans le modèle de fonctionnement traditionnel des musées, le conservateur dirige l'établissement, les visiteurs sont pris en charge dans le musée par des agents d'accueil et de surveillance et l'accompagnement proprement dit est confié à un volant de médiateurs vacataires. Comme ces derniers travaillent ponctuellement, le musée doit mettre en place un relais entre la demande des visiteurs et l'offre de travail des médiateurs. Dans une configuration minimaliste, le rôle de support organisationnel échoit à un agent d'accueil, une secrétaire ou un agent de réservation, qui se charge à la fois d'enregistrer les demandes de réservation et de proposer ces dates et horaires aux vacataires. Dans une configuration plus élaborée, un chef de service supervise une équipe d'agents d'accueil et de réservation. En général, le chef de service (ou un conservateur lorsque ce service se compose seulement d'un agent de réservation) définit l'offre d'accompagnement de manière très générale, en proposant aux visiteurs des thèmes de visite par exemple, et les médiateurs vacataires se chargent de concevoir leurs propres visites en menant des recherches bibliographiques. Ce travail de préparation représente un investissement important, car les connaissances doivent être actualisées pour chaque nouvelle exposition ou chaque nouveau thème de visite. Les heures consacrées à la préparation ne sont pas rémunérées, mais sont

9/ Leur rôle consiste à initier et entretenir des relations suivies avec les établissements scolaires des environs, pour réaliser des projets pédagogiques en partenariat par exemple. 
censées être prises en compte par une rémunération horaire élevée pour les visites ${ }^{10}$.

Le recours à des vacataires obère la nature et la diversité de l'offre d'accompagnement. Plus l'équipe de médiateurs vacataires est importante, plus le service des publics a en effet tendance à se concentrer sur la gestion de ce personnel, c'est-à-dire sur l'organisation de leur travail. L'équipe de médiateurs étant entièrement composée de vacataires rémunérés pour les seules visites, le développement et la conception des projets, la prospection des nouveaux publics sont en outre négligés faute de main d'œuvre. Le risque est que l'offre du musée ne soit ni renouvelée ni enrichie et que la demande stagne ou se concentre sur les publics captifs.

Le système des vacations ne facilite enfin pas systématiquement l'organisation de l'accompagnement, car les vacataires ne sont pas toujours disposés à étendre leur capacité d'intervention en investissant dans de nouveaux thèmes de visites, et ce d'autant qu'ils ne sont pas entièrement disponibles pour leur employeur. L'emploi à la vacation ne s'assortit en effet d'aucun engagement des deux parties (employeur et salarié) : l'employeur peut ne pas fournir de travail au médiateur vacataire, ce dernier étant en retour libre de refuser certaines propositions. Dans les services des publics, les agents de réservation doivent alors relever le défi d'apparier toutes les demandes des visiteurs à un médiateur, ce qui signifie passer une grande partie de leur temps à contacter les vacataires un par un et négocier la prise en charge de toutes les demandes, sans garantie de réussite. Le système des vacations ne tient donc pas forcément ses promesses en matière de flexibilité du travail pour les organisations muséales.

La stratégie flexible est particulièrement représentative d'un usage d'une palette contractuelle diversifiée censée optimiser la réactivité face aux demandes des clients. D'un côté, les formes particulières d'emploi assurent aux musées publics un certain degré de flexibilité, à la fois fonctionnelle et financière ; de l'autre, elles offrent des débouchés et des solutions d'attente précieuses à un ensemble hétéroclite d'individus dont le dénominateur commun est la volonté de travailler tout en préservant du temps pour une composante importante de leur identité sociale, extérieure à l'emploi de

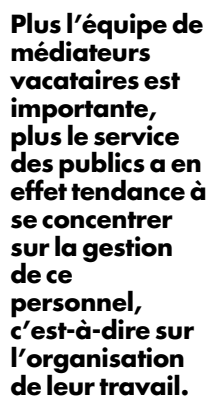

de leur travail. 
médiateur. Mais si cette situation instable peut tout à fait satisfaire les médiateurs à un moment précis de leur trajectoire, elle peut devenir intenable sur le long terme, lorsque les ressources extérieures à l'emploi font défaut. Quelles stratégies d'adaptation les médiateurs vacataires élaborent-ils en réponse aux stratégies de flexibilité des employeurs?

\section{LES STRATÉGIES DES MÉDIATEURS, UN DÉFI POUR LES STRATÉGIES DES EMPLOYEURS}

Deux tiers des emplois d'accompagnement sont des emplois non permanents, qui impliquent une rémunération aléatoire, puisque basée sur un volume de travail non fixé par contrat et généralement faible (quelques heures par semaine). Sauf exception, les vacations ne constituent pas vraiment un tremplin vers un emploi stable de médiateur et de nombreux musées fonctionnent traditionnellement avec un volant de médiateurs vacataires «fidélisés ». Ceux qui ont des ressources extérieures à l'emploi (aide familiale, revenu du conjoint...) et des compartiments biographiques distincts du travail (famille, pratique artistique, études...), peuvent y trouver leur compte, mais les autres sont en situation précaire. Ces derniers cherchent alors à réduire les risques auxquels ils sont exposés par ce système flexible (variabilité des revenus, inactivité ou perte d'emploi) en multipliant les employeurs. Ces stratégies d'ajustements et arrangements sous contrainte perturbent en retour les modes de gestion des ressources humaines des employeurs territoriaux.

\section{COMMENT RESTER MÉDIATEUR QUAND ONEST VACATAIRE ?}

Les stratégies d'organisation et de gestion flexible de la main d'œuvre muséale mettent en place une offre de travail caractérisée par une association paradoxale entre intermittence des relations d'emploi et récurrence des relations de travail. Si les vacations représentent un système d'emploi idéal pour certains médiateurs, ce système n'est pas forcément valable sur le long terme. On ne reste pas étudiant éternellement et la phase d'insertion, si elle a tendance à s'allonger pour les jeunes entrants sur le marché du travail (Céreq, 2003), doit tout de même aboutir à une stabilisation.

Frida, médiatrice dans un musée d'art de la région Rhône-Alpes, se rappelle très bien les cinq années durant lesquelles elle a été 
vacataire: "Il faut sans arrêt jongler avec l'argent, parce que la paie n'est pas fixe à la fin du mois. Donc mettons: pour neuf heures tu vas avoir $4000 \mathrm{~F}(610 €)$ de paie, et puis d'autres fois tu vas avoir $1500 \mathrm{~F}$ (229 €) parce qu'il y a quinze jours de vacances... C'est le plus pénible. [...] J'étais très raisonnable, je ne dépensais pas, je reportais, mais c'était très pénible de toujours devoir faire les comptes...». Après trente ans, les médiateurs vacataires souffrent beaucoup plus de l'instabilité de l'emploi et connaissent l'angoisse des lendemains : la précarité statutaire est en effet non seulement synonyme de protection partielle en matière de chômage, mais encore de moindre cotisation pour le système de retraite. De plus, ce système fonctionne aussi grâce aux ressources conjugales des médiateurs, qu'ils soient hommes ou femmes, et tout accident de parcours dans la vie privée peut faire basculer les vacataires dans la précarité.

L'ancienneté augmentant, les médiateurs attendent de l'institution qui les emploie une forme de reconnaissance: ils espèrent voir récompenser leur fidélité et l'accroissement de leur compétence (lié à l'expérience) par un accroissement du volume de travail ou une pérennisation du contrat. Il n'est en outre pas rare que l'accompagnement se révèle être un travail épanouissant et enrichissant, poussant les médiateurs à chercher à pérenniser ce qui était au départ conçu comme un emploi de transition. Les données du problème changent alors et les médiateurs se demandent comment continuer à exercer ce métier, alors que la majeure partie des emplois proposés est constituée d'emplois non permanents.

Juridiquement, rien n'empêche la pérennisation d'une relation de travail entre un médiateur vacataire et son employeur. La récurrence des relations de travail, stratégie bien connue des intermittents du spectacle (Coulangeon, 2004 ; Bense, 2006), apporte une certaine stabilité biographique aux médiateurs et permet à leurs employeurs d' « économiser » les coûts de recherche, de recrutement et de mise au travail (François, 2003) de nouveaux médiateurs. Les vacataires se différencient cependant des intermittents du spectacle, car ils ne cotisent pas au régime d'assurance chômage.

Les médiateurs mettent donc en place une autre stratégie très répandue parmi les intermittents du spectacle: ils multiplient les employeurs pour diversifier les risques d'activité de l'un à l'autre ${ }^{11}$, ce qui multiplie aussi leurs chances de se stabiliser dans l'une ou

11/ L'adaptation de la notion économique du portefeuille d'activité aux individus a été importée en France par Pierre-Michel Menger $(1989,1994)$ pour expliquer la combinaison des sources de rémunération dans le secteur artistique.

\section{L'ancienneté augmentant, les médiateurs attendent de I'institution qui les emploie une forme de reconnaissance.}


l'autre de ces relations d'emploi. La plupart des grandes villes françaises étant riches en établissements patrimoniaux, il est assez courant qu'un médiateur travaillant dans l'un d'entre eux travaille parallèlement dans un autre (géré ou non par la même collectivité). Imène, 35 ans, illustre bien cette démultiplication des engagements: en cumulant des contrats ponctuels ou réguliers auprès de six employeurs publics, elle tente d'atteindre un équivalent temps plein qui lui permettrait de vivre "correctement». Le cas d'Imène est extrême par la quantité d'employeurs impliquée, mais il est particulièrement exemplaire de la démarche et du rythme de constitution du portefeuille d'employeurs.

Elle a commencé à travailler en 1994 pour deux musées. Dans le premier, un musée géré par l'État, elle a été recrutée sur un contrat à durée déterminée à temps variable : elle y travaille à la demande et est rémunérée à la tâche. Dans le second, un musée municipal d'une région voisine, elle a d'abord été vacataire avant d'obtenir un contrat à durée déterminée à temps partiel, pour trois ans. En 1995, Imène a dû ajouter un établissement à ces deux premiers employeurs, car elle ne travaillait pas assez: elle a donc commencé des vacations dans un autre musée géré par l'État. Depuis 2002 enfin, elle a encore enrichi la liste de ses employeurs publics en travaillant de temps en temps pour un établissement public du ministère de la Culture et plus régulièrement dans deux offices du tourisme municipaux de la région parisienne. Pour s'assurer des revenus à mi-temps pendant trois ans, Imène a dû élargir considérablement son bassin géographique d'emploi et passer deux jours par semaine dans une ville située à deux heures de transport de son lieu de résidence. Après avoir passé sans succès le concours de conservateur (deux fois) et celui d'attaché de conservation (deux fois également), elle souhaitait vraiment changer de métier, pour stabiliser sa vie conjugale et construire un projet parental : "Si j'avais une autre possibilité, je ne resterais pas conférencière. »

En changeant à nouveau de perspective, nous allons voir que ces engagements multiples, en contraignant l'emploi du temps des médiateurs, compliquent et perturbent l'organisation de l'accompagnement et, partant, le travail du service des publics.

\section{DES STRATÉGIES DES MÉDIATEURS À UNE RECONFIGURATION DE LA GESTION DE LA MAIN D'OEUVRE D'ACCOMPAGNEMENT}

Le modèle flexible semble présenter des avantages évidents en termes organisationnels (le salarié vacataire travaille à la demande) 
et en termes économiques (la rémunération des vacations est attachée à l'acte), mais il entraîne également des contraintes pour l'organisation: la flexibilité est parfois porteuse de coûts cachés et de dysfonctionnements (Beaujoint-Bellet, 2004 ; El Akremi et al., 2004).

Dans un grand musée d'histoire de la région parisienne par exemple, quatre personnes (dont deux à mi-temps) sont uniquement affectées aux réservations et chargées d'attribuer les 30 visites quotidiennes aux 25 médiateurs vacataires. Si le nombre de médiateurs est a priori suffisant pour absorber l'activité moyenne du service, en réalité les choses sont beaucoup plus compliquées : entre les artistes, les étudiants et les multiactifs, le planning des «disponibilités » des médiateurs est très morcelé. Chaque réservation entraîne au minimum deux appels téléphoniques : le premier au médiateur pressenti, pour vérifier s'il est libre, et le second au client, pour confirmer la visite. Mais bien souvent, le médiateur n'est pas joignable sur le moment ou a finalement pris un autre engagement qui le contraint à décliner la proposition et l'agent de réservation doit tenter sa chance auprès d'une autre personne. Pour pallier le manque de disponibilité des vacataires multiactifs et reconstituer un volant de main d'œuvre disponible, les musées recrutent généralement de nouveaux vacataires. Ceci peut engendrer un cercle vicieux : le nombre de médiateurs augmente tandis que le volume de travail se maintient et cette concurrence pour le travail contraint de nouveaux vacataires à la multiactivité.

$\mathrm{Au}$ bout de plusieurs années de ce régime d'emploi flexible, les médiateurs insatisfaits de leurs conditions d'emploi se retrouvent dans une configuration classique: «loyalty, exit, voice (Hirschman, 1970). Le premier choix désigne la configuration d'appariement entre un système d'emploi flexible et un médiateur qui s'en accommode, parce que sa situation conjugale et familiale le lui permet ou parce qu'il a d'autres activités qui lui procurent d'importantes ressources identitaires. Le second choix englobe les médiateurs qui cherchent à trouver un emploi plus stable en passant des concours de la fonction publique, quitte à changer de travail, de musée, voire de ville - l'employeur doit dans ce cas recruter de nouveaux médiateurs, qui seront euxmêmes confrontés au même problème au bout de quelques années. Le dernier choix enfin, plus rare, peut concerner les médiateurs qui décident de revendiquer la stabilité en faisant appel aux syndicats et en cherchant à négocier collectivement avec l'employeur.

Les revendications des médiateurs ont longtemps trouvé peu d'écho dans les musées, car les cadres d'emploi susceptibles d'accueillir des médiateurs n'ont été créés dans la fonction publique

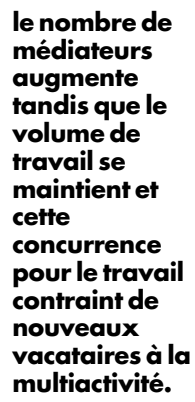

multiactivité. 
territoriale qu'en 1991. Désormais, les médiateurs vacataires peuvent donc s'appuyer sur ce nouveau cadre statutaire pour demander leur intégration dans la fonction publique; ils sont d'ailleurs aidés par les protocoles de résorption de l'emploi précaire qui, à intervalles réguliers depuis 1946, réglementent les intégrations directes et concours réservés (Plan Perben en 1995, Plan Sapin en 2001).

Dans l'un des cas étudiés, l'intégration directe des médiateurs vacataires a été possible en catégorie $\mathrm{B}$ grâce à une reconfiguration de la politique culturelle locale ${ }^{12}$ : en intégrant les médiateurs dans la fonction publique, la municipalité a redéfini les objectifs de l'accompagnement muséal et intervient beaucoup plus dans le travail des médiateurs. L'organisation du travail a elle-même été complètement repensée autour des obligations de service des médiateurs fonctionnaires. Le service des publics dispose désormais d'une offre d'accompagnement fixe (par exemple trois visites par jour et par médiateur), proportionnelle au nombre de médiateurs titularisés. Le service des publics propose donc différents créneaux horaires aux visiteurs, renversant la traditionnelle stratégie de fonctionnement : ce n'est plus au service de trouver un médiateur pour chaque demande, mais aux clients de s'adapter à un planning prédéfini. L'incertitude est donc réduite à la fois pour les salariés et pour les employeurs.

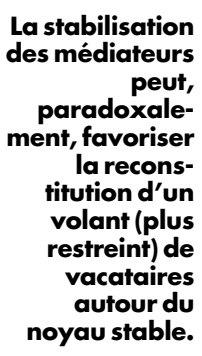

Notons cependant que la stabilisation des médiateurs peut, paradoxalement, favoriser la reconstitution d'un volant (plus restreint) de vacataires autour du noyau stable. Cela s'est produit dans l'exemple évoqué ci-dessus: quand la dizaine de médiateurs a été titularisée, ceux-ci ont dû s'absenter pour suivre des formations obligatoires. Même si le service s'est organisé pour échelonner ces départs, le planning a été considérablement perturbé. Trois nouveaux vacataires ont donc été recrutés dès la titularisation des anciens médiateurs vacataires, pour remplacer les uns et les autres durant leur formation donc, puis durant leurs congés ou vacances. Une fois le cercle périphérique reconstitué par des vacataires, l'employeur et ces nouveaux salariés se retrouvent dans la configuration initialement décrite.

Pour les employeurs, la vacation semble être la forme particulière d'emploi qui répond le mieux aux besoins de flexibilité induits par l'accompagnement; pour les salariés, cette forme d'emploi précaire offre un travail rémunéré dans un domaine de qualification pointu,

12/ Frida, citée plus haut, fait partie d'une équipe d'une dizaine de médiateurs vacataires depuis 1993 (date de l'ouverture du musée), dont la plupart a été récemment titularisée en catégorie B de la fonction publique territoriale dans le cadre de la Loi nº6-1093 du 16 décembre 1996 relative à la résorption de l'emploi précaire - ce cas reste néanmoins une exception dans les musées enquêtés par l'ampleur et l'intensité de l'engagement syndical des médiateurs. 
qui constitue une étape dans la trajectoire. Cet appariement atypique est un jeu dont l'équilibre peut se dérégler avec le temps, car ces emplois sont pourvus par des profils particuliers : être médiateur signifiant le plus souvent être employé de façon temporaire et intermittente, cette situation est surtout attractive quand l'emploi n'est pas la seule composante de l'emploi du temps et de l'identité. Pour les médiateurs « de passage » ou "à côté d'autre chose », et ceux qui y restent «bloqués », par défaut, il y a toujours, au départ, l'idée d'une phase transitoire, d'un travail d'appoint. Certaines et certains s'installent malgré tout, parfois malgré eux, dans un travail régulier sur le long terme sans que la forme d'emploi évolue, ce qui les pousse à multiplier les engagements auprès d'employeurs différents.

Du côté des employeurs, la situation se complique alors ; les médiateurs engagés comme vacataires n'étant plus aussi disponibles, le travail d'appariement entre demande de services d'accompagnement et offre de travail de la part des vacataires devient plus laborieux et aléatoire. Si l'on ajoute à cela le fort turn over qui découle de la présence d'étudiants ou de jeunes diplômés qui ne sont là que pour un temps, on comprend que les musées aient dû imaginer d'autres formes d'organisation du travail, en proposant aux médiateurs des emplois plus stables, au moins à moyen terme, tout en conservant un volant de vacataires pour les remplacements et autres impondérables.

Dans le secteur culturel comme ailleurs, la mobilité des travailleurs s'accroît avec l'instabilité des contrats (Germe, 2001) : la segmentation de l'emploi et la «flexibilisation » du travail complexifient les trajectoires professionnelles, y compris dans la fonction publique. Plus largement, confronter les usages des formes particulières d'emploi par les employeurs et par les salariés est une manière d'aborder différemment les problématiques de flexibilité et de précarité, qui pourrait renouveler l'approche de terrains et de populations abondamment étudiées comme les salariés peu qualifiés par exemple, ou plus inédites comme les salariés très diplômés du secteur privé. Les institutions culturelles captant nombre des diplômés de l'université issus de la dernière période de «massification » (Chauvel, 1998), le marché du travail des médiateurs dans les musées publics offre un premier terrain d'observation pour observer des phénomènes plus généraux affectant le marché du travail.

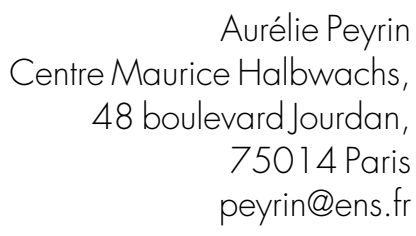


Aurélie Peyrin

\section{RÉFÉRENCES BIBLIOGRAPHIQUES}

\author{
AUBIN E., 2004 \\ BALLÉ C., POULOT D., 2005
}

BEAUJOINT-BELLET R., 2004

BENSE C., 2006

BERG C., DESMAREZ P., EDWARDS J., ENGELS X., GILEJKO L., JACQUOT L., NOSBONNE C., TOWALSKI R., WILLEMS H., 2006

BREEN R., COOKE L.P., 2005

CANCÉ R., 2002

CÉREQ., 2003

CHAUVEL L., 1998

COULANGEON P., 2004

DONNAT O., 1999

DUPUY Y., LARRÉ F.,

EL AKREMI A., IGALENS J., VICENS C, 2004

FONS J.-P., MEYER J.-L., 2005

FRANÇOIS P., 2003

FULLIN G., 2005

GERME J.-F., MONCHATRE S., POTTIER F., 2003
Droit de la fonction publique, Gualino Éditeur.

La Transformation des musées en Europe. Une mutation inachevée, Paris : La Documentation Française.

Aux sources de la flexibilité : quelles transformations?, in : Beaujoint-Bellet R. (dir.) Flexibilités et performances.

Stratégies d'entreprises, régulation, transformation du travail, Paris : La Découverte, p. 9-19.

Précarité en échange. Enquête sur l'implication au travail, Montreuil : Éditions Aux lieux d'être.

Employment in the public sector: a trans-national and European analysis, Rapport de recherche, Université Nancy 2, Grée, 2L2S.

The Persistence of the Gendered Division of Domestic Labour, European Sociological Review, vol. 21, $\mathrm{n}^{\circ} 1$, p. 43-57.

Travailler en contrat à durée déterminée, entre précarité contrainte, espoir d'embauche et parcours volontaire, Travail et emploi, no 89 , p. 29-44.

De l'enseignement supérieur à l'emploi : les trois premières années de vie active de la Génération 98.

La seconde explosion scolaire : diffusion des diplômes, structure sociale et valeur des titres, Revue de l'OFCE, $n^{\circ} 66$, p. 5-36.

Les musiciens interprètes en France. Portrait d'une profession, Paris : La Documentation Française.

Les pratiques culturelles des Français. Enquête 1997. Paris : la Documentation Française.

1998

Entre salariat et travail indépendant : les formes hybrides de mobilisation du travail, Travail et emploi, n ${ }^{\circ} 77$, p. 1-14.

Flexibilité organisationnelle. Complexité et profusion conceptuelles, in : R. Beaujoint-Bellet (dir.), Flexibilités et performances. Stratégies d'entreprises, régulation, transformation du travail, Paris: La Découverte, p. 23-50.

La flexibilité dans les fonctions publiques en Angleterre, en Allemagne et en France. Débats, enjeux, perspectives, Paris : La Documentation Française.

Définition de nouvelles normes d'emploi et organisation par projets : le cas de la musique ancienne.

Communication aux IX journées de sociologie du travail, Contraintes, normes et compétences au travail. Négociation de nouvelles normes de travail et d'emploi, Paris : Centre Pierre-Naville, p. 63-71.

Trajectoires et identités dans les emplois précaires en Italie, Formation Emploi, n 91, p. 123-135.

Les mobilités professionnelles : de l'instabilité dans l'emploi à la gestion des trajectoires, Paris, La Documentation Française. 
GERME J.-F., 2001

Grelet Y., Mansuy M., 2004

HIRSCHMAN A.O., 1970

JOIN-LAMBERT O., LOCHARD Y., Servir l'État et l'usager. Définition et indéfinition du travail par RAVEYRE M., UGHETTO P., 2004 le public dans le domaine des musées, Rapport pour le

JOURDAIN C., 2002

MENGER P.-M., 1989

MENGER P.-M., 1994

MONIOLLE C., 1999

MOULIN R., QUEMIN A., 1993

OCTOBRE S., 2001

PETIT H., 2002

PETIT H., 2005

PEYRIN A., 2005

POULARD F., 2005

SCHNAPPER D., 1989

TOBELEM J.-M., 2005 ministère de la Recherche et le Centre national de la fonction publique territoriale, IRES.

Au-delà des marchés internes : quelles mobilités, quelles trajectoires ?, Formation Emploi, n 76 , p. 129-145.

De la précarité de l'emploi à celle des trajectoires : une analyse de l'insertion en évolution, Formation Emploi, $n^{\circ} 85$, p. 87-99.

Exit, Voice, and Loyalty : Responses to Decline in Firms, Organizations, and States, Cambridge : MA : Harvard University Press.

Intérimaires, les mondes de l'intérim, Travail et emploi, n 89 , p. 9-28.

Rationalité et incertitude de la vie d'artiste, L'année sociologique, vol. 39, p. 111-151.

Appariement, risque et capital humain : l'emploi et la carrière dans les professions artistiques, in : Menger P.-M. et Passeron R., L'art de la recherche. Essais en l'honneur de Raymonde Moulin, Paris : la Documentation Française, p. $219-238$

Les agents non titulaires de la fonction publique de l'État : entre précarité et pérennité, Paris : Librairie générale de droit et de jurisprudence.

La certification de la valeur de l'art. Experts et expertises, Annales, vol. 48, n 6, p. 1421-1445.

Construction et conflits de la légitimité professionnelle : qualification et compétences des conservateurs de musées, Sociologie du travail, $\mathrm{n}^{\circ} 43$

Fondements et dynamique de la segmentation du marché du travail. Une analyse sur données françaises, Thèse de doctorat, Université Paris 1 Panthéon-Sorbonne.

Du constat de précarité généralisée à l'hypothèse d'une segmentation persistante. Le cas de la France. Journées Approches du marché du travail, LEST - Groupe de recherche en économie et sociologie, Aix-en-Provence, 29 et 30 septembre.

Faire profession de la démocratisation culturelle. Travail, emploi et identité professionnelle des médiateurs de musées, Thèse de doctorat, EHESS.

Les musées de collectivités, les conservateurs et la mise en cuvre des politiques culturelles, Thèse de doctorat, Université Paris 1.

Rapport à l'emploi, protection et statuts sociaux. Revue Française de Sociologie, vol. $30, n^{\circ} 1$, p. 3-29.

Le nouvel âge des musées. Les institutions culturelles au défi de la gestion, Paris : Armand Colin. 